\title{
Numerical Modeling of Economical Market Forces for an Intelligent Delivery of Dynamical Optimal Impact Solution (DOI)
}

\author{
${ }^{1}$ Mahmoud Iskandarani and ${ }^{2}$ Ghaleb El-Refae \\ ${ }^{1}$ Faculty of Science and Information Technology, ${ }^{2}$ Faculty of Economics and Administrative Sciences \\ Al-Zaytoonah University of Jordan, P.O.Box 911597, Post Code 11191, Amman, Jordan
}

\begin{abstract}
An intelligent model for the purpose of evaluating and affecting delivery of services as a function of initial capital investment is developed and discussed. The algorithm associated with such a model, maps the principles of sustainability of business success as dependent not only on the content of the business but also on the impact that it has on the beneficiaries and users of its outputs. Such modeled functions take into account surrounding factors and diverse forces that influence the delivery of services, goods or in general any desired output. An evidence of accumulative market forces and their impact on optimal delivery of goods and services is proved and illustrated. The obtained curves proved that the general collective effect of surrounding market forces is power based, which does agree with witnessed practical cases. Using intelligence in the model greatly assisted in determining and predicting vital critical points and breaking points of optimal service delivery.
\end{abstract}

Key words: Knowledge base, economics, optimal impact, optimal knowledge, decision making, modeling

\section{INTRODUCTION}

For a long time, economics has recognized two main factors of production as main contributors: labor and capital. This is now changing. Information and knowledge are replacing capital and energy as the primary wealth-creating assets, just as the latter two replaced land and labor many years ago. In addition, technological developments have transformed the majority of wealth-creating work from physically based to "knowledge-based." Technology and knowledge are now the key factors of production [6, 7, 18, 21, 22, 26]. With increased mobility of information and the global work force, knowledge and expertise can be transported instantaneously around the world, and any advantage gained by one company can be eliminated by competitive improvements overnight. The only comparative advantage a company will enjoy will be its process of innovation by combining market and technology know-how with the creative talents of knowledge workers to solve a constant stream of competitive problems with an ability to derive value from information. We live today in an information society with a knowledge economy where knowledge management is essential. The sole determination of sustained business success and the singular cause of business failure is knowledge and/or information content based. Business optimization has proven to be possible only from a basis of "informed choice" and economic knowledge differentials. Optimal Impact policy is one, which causes the critical choices to be made consistent with maximizing goal gains. A traditional optimal policy has the property that whatever the initial state and initial choices are, the remaining choices must constitute an optimal policy with respect to the state resulting from the original choices $[1,2,3,4,5]$.

The previous implies that actual achievement of goal is open to all starting points or current states. However it is the absolute requirement to correctly measure the actual current "state" of the success ingredients, which are information differentials. Information content (knowledge) creates measurable bias toward better choices; hence, Capturing or achieving an economic utility function requires that the goal be able to be measured. A metric guidance system is absolutely required for the determination that the choices are properly goal directed. Navigation fails when the feed forward and feedback measures are faulty.

In this paper an optimal knowledge numerical model is presented. The model will measure the interaction between various market forces under different conditions and will provide an indication to the status of the market. Such information will assist in decision making in order to achieve optimal delivery of contents, and is used as part of an intelligent approach to optimal knowledge and its optimal impact on deliverable economical variables such as optimal asset allocation in an enhanced index product. 
Background: As technology advances and becomes more complex, it becomes almost impossible for any professional to ignore other influences and forces that affect a certain discipline. Two factors should be considered and implemented in a model are:

(I) The mechanism for achieving goals

(II) Humans implementing such mechanism.

To apply solutions that provide an impact effect, better understanding of such inhomogeneous market should be reached. Two sources of errors are important and need to be considered:

(I) Operational errors

(II) Lack of knowledge errors

It is essential to implement knowledge in business, which will account for different types of control and requirements that reaches beyond the local scope to cover an international range. Hence, it is essential to integrate the effect of various market forces to obtain the overall effect on a certain market. This leads to complex modeling.

Knowledge economy requires the use and implementation of knowledge management, which forces the use of various technological approaches and techniques. Such heavy task requires the maximization of intellectual capital to sustain a knowledge driven economy. This will certainly affect the overall performance of the managed economy.

Model Description: In our Model, shown in figures 1 and 2 not only the attributes of the individual inputs are considered but also their mutual and correlative features among them and in relation to a Dynamical Optimal Impact (DOI). Our model assumes that there is more than one "state" which might characterize the modeled variable. As the market changes with influencing factors which are sometimes unexpected and unpredictable, the market reaction to such factors maybe systematic that would not show any kind of movement or dynamical change as a function of such affecting variables [27], hence the outcome of such changes would not be identified and controlled to deliver an optimal impact. Hence, providing such a simulative algorithm is very useful to dynamically track such changes. The numerical analysis presented here using finite element analysis. This analysis modeled such interactive market forces in twodimensions, using our specifically developed complex mathematical expression given by:

Field Strength from Market Forces

$=\left[\sum_{n=1}^{i}\left[\left(\frac{P_{n-1}-P_{n}}{\pi}\right)\left(\frac{\left(x-d_{n}\right)}{\left(x-d_{n}\right)^{2}+y^{2}}\right)\right]+\left[\left(\frac{P_{n-1}-P}{\pi}\right)\left(\frac{y}{\left(x-d_{n}\right)^{2}+y^{2}}\right)\right]\right]^{1 / 2}$

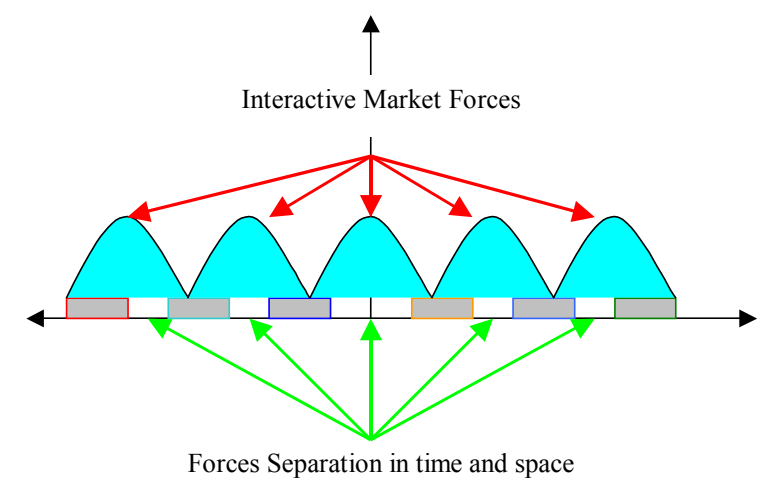

Fig. 1: Illustration of interactions in the market

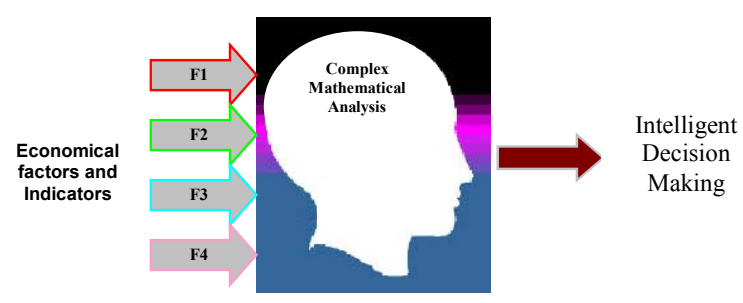

Fig. 2: Decision making process for interacting market forces

Where, P: Force Potential, d: Separating Space

The resulted data from the numerically modelled complex function is used as input to an intelligent classification system as illustrated in figure 3 below.

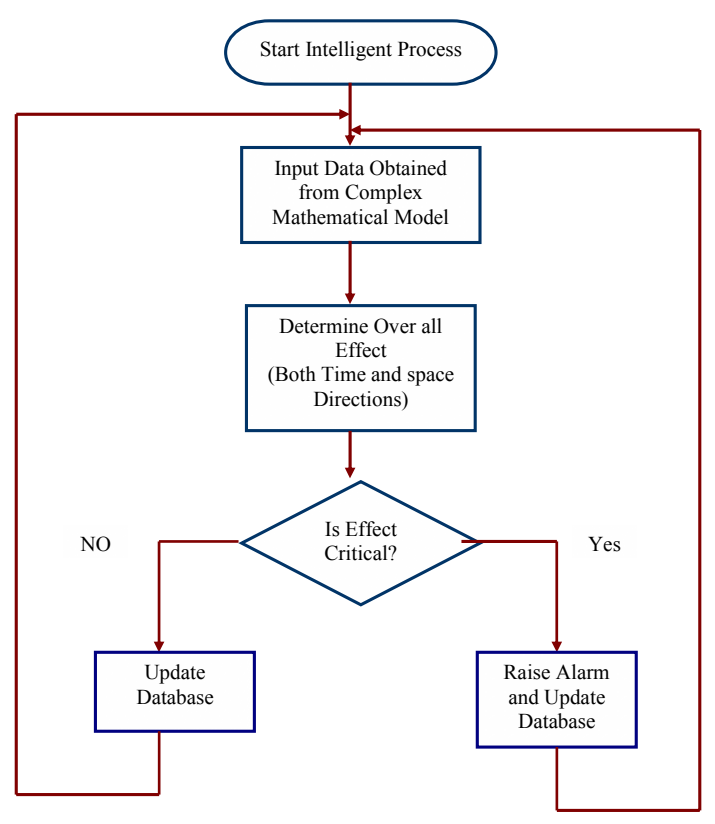

Fig. 3: Intelligent Decision Making 
Analysis, Discussion, and Conclusions: Figures 4 and 5 illustrate the numerically simulated complex mathematical expression, which is developed to account for multi-force interaction in a homogeneous economical environment. As an approximation method; when used; the continuous field with infinite degrees of freedom is replaced with a discrete one with a finite number of degrees of freedom. When applied, the method subdivides the continuous field into a finite number of areas called ELEMENTS which are interconnected at finite points called NODES at which the boundary conditions are specified. The solution is then approximated over the whole structure of elements maintaining continuity at each node. The most effective solutions obtained from finite element software are achieved by combining different operations involved in the complete process.

Two important variables considered for the shape functions used to form the finite element mesh:

(a) NKTP: Specifies the element type.

(b) NORDER: Specifies the element shape.

Two types of shape function elements are used in the analysis separately:

(1) 4-node element with NKTP $=102$, NORDER $=1$, for initial result (Fast with low memory usage)

(2) 8-node element with NKTP $=102$, NORDER $=4$, for accurate representation (Slow with high memory usage)

Figure 4 illustrates the accumulative effect of such forces on a selected area, whereby, we can see the power like increase of field strength that an affected point will feel as a result of forces interacting. Such forces would reach a specific steady state dictated through each force contribution. Such a state is only steady and stable under certain economical circumstances, hence, will change and vary if economical market surrounding changes to reach another steady state and so on. This dynamical behavior is an efficient way of measuring and predicting market movements and hence will contribute towards delivering of optimal economical solutions. Figure 5 complements the overall view by showing a different dimensional space that measures the effect of interacting forces as a function of force ability and reaching effect, which could be affected by general local, and global economical activities. Both figures illustrate the convergence and divergence mechanism for the describing complex function and its simulation of the interacting forces to a steady state as a result of various field effects in time and space. The developed model and algorithm incorporates an optimal policy that functions on the principles of the initial state and initial choices producing future results that are not totally based on the initial values as normally the case is, but dynamically and intelligently adaptable to ensure optimal investment and maximum satisfaction for both manufacturer and consumer, user and service provider, university and students, clients and servers. Such adaptability and dynamism is achieved using complex mathematical analysis that accounts for various interacting market forces. Such a novel mathematical model, which is developed and discussed in this paper proved to be an effective tool in describing the overall effect of interacting economical factors under different conditions to deliver the impact knowledge objectives under moving market variables.

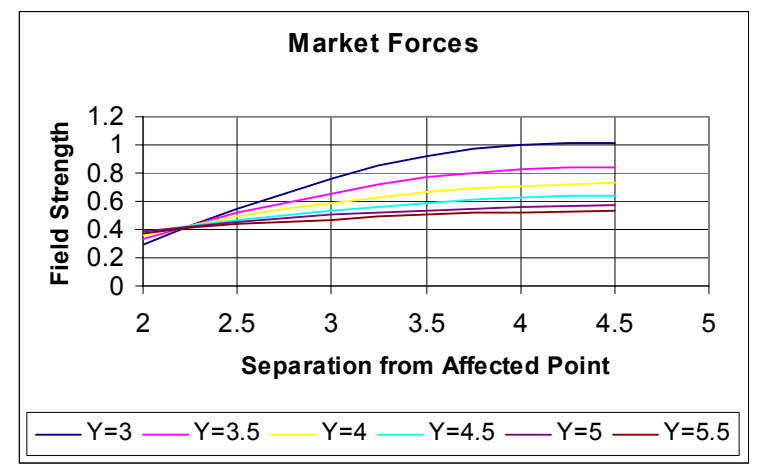

Fig. 4: Accumulative effect of market forces (xspace)

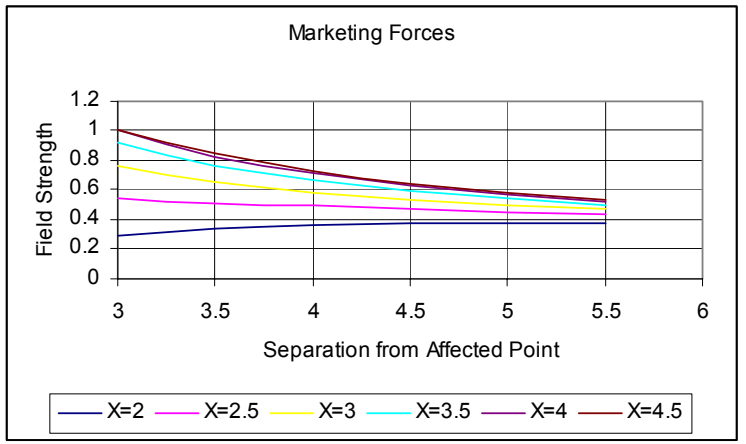

Fig. 5: Accumulative effect of market forces ( $y$ space)

\section{REFERENCES}

1. Tesfatsion, L., 2001. Agent-based computational economics: Growing economies from the bottomup. Artificial Life, 8: 55-82.

2. Aby, C., 1995. Asset management for pension plans: An intermarket approach using Technical Analysis. J. Pension Planning \& Compliance, 21: 44-64.

3. Lettau, M., 1997. Explaining the facts with adaptive agents: The case of mutual fund flows. J. Economic Dynamics and Control, 21: 1117-1148.

4. Vriend, N., 2000. An illustration of the essential difference between individual and social learning and its consequences for computational analysis. J. Economic Dynamics and Control, 24: 1-19.

5. Brock, W., J. Lakonishok and B. LeBaron, 1992. Simple technical trading rules and the stochastic properties of stock returns. J. Finance, 47: 17311764. 
6. Keith, H.T.M., 2004. Regional Wage and Employment Responses to Market Potential in the EU. S'eminaire Roy.

7. Brock, W. and C. Hommes, 1998. Heterogeneous beliefs and routes to chaos in a simple asset pricing model. J. Economic Dynamics and Control, 22: 1235.1274.

8. Chiarella, C. and X. He, 2001. Asset pricing and wealth dynamics under heterogeneous expectations. Quantitative Finance, 1: 509-526.

9. Chiarella, C. and X. He, 2002. Heterogeneous beliefs, risk and learning in a simple asset pricing model. Computational Economics, 19: 95-132.
10. LeBaron, B., W.B. Arthur and R. Palmer, 1999. Time series properties of an artificial stock market. J. Economic Dynamics and Control, 23: 14871516.

11. Raberto, M., S. Cincotti, S. Focardi and M. Marchesi, 2001. Agent-based simulation of a financial market. Physica A, 299: 320-328.

12. Mahmoud, I. and G. El-refae, 2005. Modeling of economical market forces using neural based dynamical optimal knowledge evaluation algorithm. The 5th Intl. Conf., Knowledge Economy and Economical Development, 27-28 April 2005, Alzaytoonah University of Jordan. 